2016/17

Entry in First-price Auctions with Signaling

Olivier BOS AND TOM TRUYTS

\title{
50 YEARS OF
}

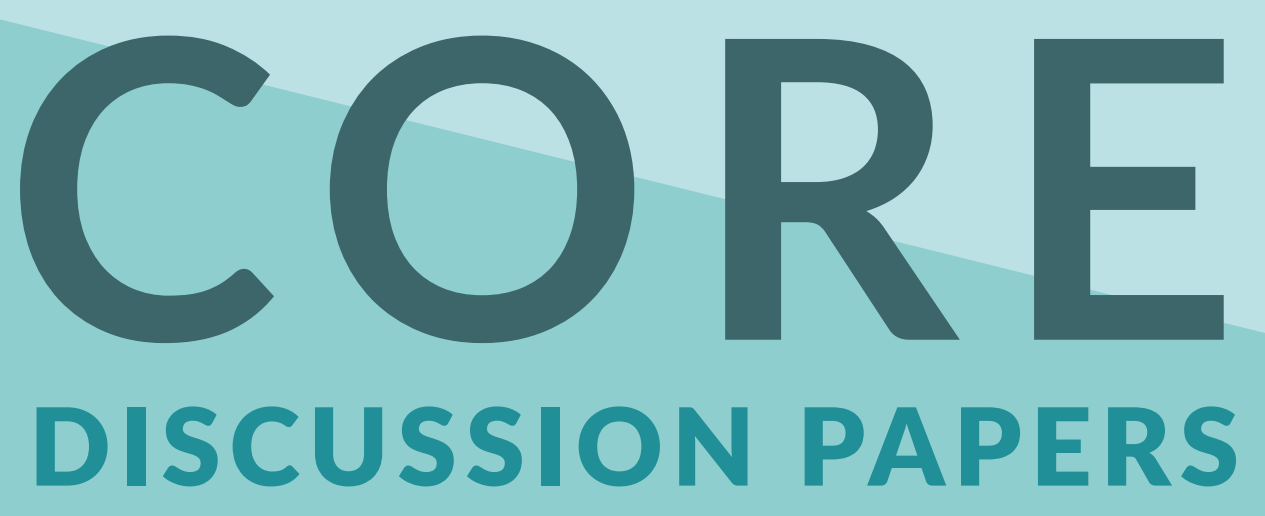




\section{CORE}

Voie du Roman Pays 34, L1.03.01

Tel (32 10) 474304

Fax (32 10) 474301

Email: immaq-library@uclouvain.be

http://www.uclouvain.be/en-44508.html 


\title{
Entry in First-price Auctions with Signaling
}

\author{
Olivier Bos*and Tom Truyts ${ }^{\dagger}$
}

\begin{abstract}
We study the optimal entry fee in a symmetric private value first-price auction with signaling, in which the participation decisions and the auction outcome are used by an outside observer to infer the bidders' types. We show that this auction has a unique fully separating equilibrium bidding function. The expected revenue maximizing entry fee is the maximal fee that guarantees full participation.

JEL: D44; D82

Keywords: Monotonic signaling; social status; first-price auction, entry
\end{abstract}

\section{Introduction}

In many auction settings, participants care about the information that their performance in the auction discloses to others, e.g., to other market parties, to the media or to the general public. Giovannoni and Makris (2014) study how the outcome of a take-over auction can serve as a

*Panthéon-Assas University, LEMMA, 4 rue Blaise Desgoffe, 75006 Paris, France. E-mail: olivier.bos@u-paris2.fr, tel.: +33170232005.

Olivier Bos gratefully acknowledges financial support from the ANR DBCPG. This research has been conducted as part of the Labex MME-DII (ANR11-LBX-0023-01) project.

${ }^{\dagger}$ CEREC, Saint-Louis University - Brussels, Boulevard du Jardin botanique 43, 1000 Brussels, Belgium; Center for Economic Studies, University of Leuven; CORE, Université Catholique de Louvain. E-mail: tom.truyts@usaintlouis.be, tel.: +3222117845 .

Tom Truyts gatefully aknowledges financial support from the Belgian French speaking community ARC project $n^{\circ} 15 / 20-072$, "Social and Economic Network Formation under Limited Farsightedness: Theory and Applications", Université Saint-Louis Bruxelles, October 2015 - September 2020. 
signal of management quality, in function of a post-auction job market for managers. Goeree (2003) shows how the outcome of a single license technology auction can reveal information to competitors about the importance of the the new technology's cost reduction for the auction's winner, in function of a post-auction Cournot game. ${ }^{1}$ Bos and Truyts (2014) consider charity and art auctions in which bidders care about how the general public perceives their altruism or wealth. In all these examples, an outside observer uses the auction outcome to infer the private information of the bidders, and the bidders strategically adapt their bidding strategies in function of the additional signaling game implied in these auctions. Giovannoni and Makris (2014) show that, in the presence of signaling concerns, the auction's expected revenue depends on the information that the auctioneer shares with the outside observer - in addition to the winning bidder's identity -, and which is either 1) none of the bids, 2) only the highest bid, 3) only the second highest bid or 4) all bids. Bos and Truyts (2014) study independent private value auctions in which the outside observer sees the identity and payment of the winning bidder. They obtain a strict ranking of different auction formats in terms of expected revenue: the first-price and all-pay auctions dominate the English auction, but are dominated by the second-price auction.

Entry is considered exogenously given in the above papers, but potentially gives bidders an additional instrument to distinguish themselves from worse types in the context of auctions with signaling, if the outside observer observes the bidders' payments and the winner's identity, i.e., the entry fee paid by each participating bidder, the winner's identity and the winner's payment. The auctioneer can exploit the value of this additional signaling instrument to bidders in order to raise additional revenue.

In this paper we study an independent private value first-price auction with entry and linear payoff functions, in a setting similar to Bos and Truyts (2014). We assume that the bidders care about three things. The first two are standard: their payment and the prize if they win. In addition, the bidders care about the expected value of the outside observer's beliefs about their type. We characterize the fully separating bidding equilibrium, and show that the expected revenue maximizing entry fee is the maximal fee that guarantees full participation. Hence, the auctioneer does not set an entry fee that would allow a strict subset of bidders to distinguish themselves from worse types, but rather

\footnotetext{
${ }^{1}$ Other analyses of auctions with signaling in function of an aftermarket in industrial organization applications include Das Varma (2003) and Katzman and RhodesKropf (2008).
} 
uses the fee to ensure that the outside observer holds, in equilibrium, the worst possible beliefs concering a non-participating bidder. This maximal punishment for non-entry in terms of the outside observer's inferences allows the auctioneer to extract a sizable entry fee from all bidders with probability one, which maximizes the auction's expected revenue. We also comment on the equivalent use of entry fees in other auction formats with signaling.

Entry fees are commonly used and analyzed as instruments to improve the revenue performance of auctions. Levin and Smith (1994) show that positive entry fees maximize the expected revenue in every mechanism. More recently, Janssen et al. (2011) investigate a two-step auction game: first, bidders choose a publicly announced individual entry fee, and next, each bidder participates in the auction. Interestingly, this two-step auction in which bidders signal by means of the individual entry fees restores efficiency, despite negative externalities.

The paper is organized as follows. Section 2 introduces the formal setting. Section 3 characterizes the equilibrium and presents the main results. Finally, Section 4 briefly discusses other auction formats with entry and signaling. All proofs are collected in a mathematical appendix.

\section{Formal Setting}

Consider $n$ bidders, indexed $i$, bidding for a single object which is allocated by means of an auction to the highest bidder. Bidder $i$ 's valuation for the object (her 'type'), is denoted $V_{i}$, and is assumed i.i.d. and drawn according to a $C^{2}$ distribution function $F$ with support on $[\underline{\mathrm{v}}, \bar{v}] \subset \mathbb{R}_{+}$. Let $f \equiv F^{\prime}$ denote the density function. Bidder $i$ 's realization of $V_{i}$, denoted $v_{i}$, is her private information, but the number of bidders and the distribution $F$ are common knowledge.

To participate in the auction, a bidder pays an entry fee $\varphi \in \mathbb{R}_{+}$, chosen by the auctioneer, and submits a non-negative bid. As all bidders share the same beliefs about other bidders' valuations, they are assumed to follow a symmetric entry and bidding strategy. The entry strategy is denoted $e:[\underline{\mathrm{v}}, \bar{v}] \rightarrow\{0,1\}$, with $e(v)=1$ indicating that a $v$ type bidder pays the fee $\varphi$ to participate in the auction, and the bidding strategy is denoted $\beta:[\underline{\mathrm{v}}, \bar{v}] \rightarrow \mathbb{R}_{+}$. Finally, let $\mathbf{e}$ be the vector of entry decisions and let $\boldsymbol{b}=\boldsymbol{\beta}(\boldsymbol{v})$ denote the vector of bids given a vector of valuations $\boldsymbol{v}$, with $b_{i}$ the effective bid of $i$-th bidder. An auction mechanism maps a pair vectors describing the entry-decisions and the bids $\boldsymbol{b}$ to a winner, denoted $i^{*}$, and payments $\boldsymbol{p}$.

Apart from the auction's outcome, bidders also care about the beliefs 
that an uninformed party, the 'receiver', has about their type. This receiver can represent, e.g., the general public or press, business contacts or acquaintances of the bidder, or experts related to the object sale. The receiver is assumed to observe the entry decisions of each bidder, the auction's winner and the winner's payment $\left(\mathbf{e}, i^{*}, p_{i^{*}}\right)$. The receiver's beliefs, denoted $\mu$, are a probability distribution over the type space, such that $\mu_{i}\left(v \mid\left(\mathbf{e}, i^{*}, p_{i^{*}}\right)\right)$ is the probability of bidder $i$ being of valuation type $v$ given $\left(\mathbf{e}, i^{*}, p_{i^{*}}\right)$. Let $\mu\left(\mathbf{v} \mid\left(\mathbf{e}, i^{*}, p_{i^{*}}\right)\right)$ then be a probability distribution over vectors of valuations $\mathbf{v}$ given $\left(\mathbf{e}, i^{*}, p_{i^{*}}\right)$. The receiver's beliefs are (Bayesian) consistent with an entry strategy $e$ and a bidding strategy $\beta$ if

$$
\mu\left(\boldsymbol{v} \mid\left(\mathbf{e}, i^{*}, p\right)\right)=\frac{\operatorname{Pr}\left(\mathbf{e}, i^{*}, p_{i^{*}} \mid \mathbf{e}(v), \boldsymbol{\beta}(\boldsymbol{v})\right) \prod_{i} f\left(v_{i}\right)}{\int \operatorname{Pr}\left(\mathbf{e}, i^{*}, p_{i^{*}} \mid \mathbf{e}\left(v^{\prime}\right), \boldsymbol{\beta}\left(\boldsymbol{v}^{\prime}\right)\right) \prod_{i} f\left(v_{i}^{\prime}\right) \boldsymbol{d} \boldsymbol{v}^{\prime}} .
$$

The utility of bidder $i$, given an auction outcome $\left(i^{*}, \mathbf{p}\right)$, consists of two parts. The first part is standard: the value for the object for the winner of the auction, minus the payment, consisting of the entry fee and, for the winner, the payment of his own bid. The second part is the expected value of the receiver's beliefs about bidder $i$ 's type given $\left(\mathbf{e}, i^{*}, p_{i^{*}}\right)$, denoted $E\left(V_{i} \mid \mu_{i}\left(V_{i} \mid \mathbf{e}, i^{*}, p_{i^{*}}\right)\right)$ :

$u_{i}\left(v_{i}, p_{i} \mid \mu_{i}\right)=\left\{\begin{array}{cc}v_{i}-p_{i}-\varphi+E\left(V_{i} \mid \mathbf{e}, i^{*}, p_{i^{*}}\right) & \text { for winner } i=i^{*} \\ -\varphi+E\left(V_{i} \mid \mathbf{e}, i^{*}, p_{i^{*}}\right) & \text { for participating loser } i \neq i^{*} \\ E\left(V_{i} \mid \mathbf{e}, i^{*}, p_{i^{*}}\right) & \text { for non-participating loser } i \neq i^{*}\end{array}\right.$

As in Bos and Truyts (2014), this utility function either represents a psychological game, in which bidders care directly about the receiver's beliefs, as humans care about the good opinion of others, or it is a reduced form of a game in which the receiver chooses an action given her beliefs, while the bidders care about this action.

We study the symmetric perfect Bayesian equilibria (PBE) of this auction game with signaling. A PBE is then described by a pair of strategies and beliefs $(e, \beta, \mu)$ such that:

1. The entry and bidding strategies $(e, \beta)$ maximize the expected utility for all types $v$, given that all other bidders play $(e, \beta)$ and given the receiver's beliefs $\mu$

2. The receiver's beliefs $\mu$ are Bayesian consistent with the strategies $(e, \beta)$, as in $(1)$.

As in Bos and Truyts (2014) and Giovannoni and Makris (2014), we apply the D1 criterion of Banks and Sobel (1987), which refines the set of equilibria by restricting out-of-equilibrium beliefs, in order 
to avoid the usual equilibrium multiplicity of signaling games. The D1 criterion restricts out-of-equilibrium beliefs by considering which bidder types are more likely to gain from an out-of-equilibrium bid, compared to their equilibrium expected utility. More precisely, if the set of beliefs for which a bidder gains from a deviation to an out-of equilibrium bid $b$ (w.r.t. her equilibrium expected utility) is larger for one bidder type than for another, then the D1 criterion requires out-of-equilibrium beliefs to attribute zero probability to the latter type having deviated to $b$. In the present context, the D1 criterion imposes a certain monotonicity on out-of-equilibrium beliefs: if a certain bidder type $v$ makes a certain bid, then a strictly higher out-of-equilibrium bid should not be attributed to a bidder type lower than $v$, and if no bidder type pays the entry fee in equilibrium, then a bidder who deviates to paying the entry fee should be interpreted as the highest bidder type.

\section{Equilibrium Analysis}

We focus on symmetric perfect Bayesian equilibria with a strictly increasing bidding function, and in which the entry decision is monotonic w.r.t. types in the sense that there exists at most one cut off type, denoted $\tau \in[\underline{\mathrm{v}}, \bar{v}]$, such that all bidder types with a valuation above $\tau$ choose to pay the entry fee $\varphi$ in equilibrium, and that all the bidders with a valuation below $\tau$ prefer to stay out.

Let us then first consider the problem of a type $v$ bidder who wishes to pay the entry fee $\varphi$ in order to participate in the auction. If the PBE is fully separating, then the type of the bidder who wins the first price auction is fully revealed to be $\beta^{-1}\left(\beta\left(v_{i^{*}}\right)\right)=v_{i^{*}}$ in equilibrium. If the auction's winner is of type $v_{i^{*}}$ and if only the bidders with a valuation above $\tau \leq v_{i^{*}}$ decide to participate, then all losing participating bidders

are estimated to be of type $\frac{\int_{\tau}^{v_{i}^{*}} x d F(x)}{F\left(v_{i^{*}}\right)-F(\tau)}$. However, in the contingency that the type $v$ bidder does not win the auction, he ex ante does not know the type of the winner, except that the winner must have a higher valuation than his. Therefore, the type $v$ bidder takes the expectation over the winning bidder's type, conditional on the fact that it is higher than his. As such, the expected value of the receiver's beliefs about the $v$ type bidder in case of losing the auction and an entry cut off type $\tau$ is

$$
\frac{1}{1-F^{n-1}(v)} \int_{v}^{\bar{v}} \frac{\int_{\tau}^{y} x d F(x)}{F(y)-F(\tau)} d F^{n-1}(y) .
$$

Finally, if the bidding function is strictly increasing, then the $v$ bidder's probability of winning the auction is equal to the probability of the $n-1$ other bidders having a valuation lower than $v$, i.e., $F^{n-1}(v)$. 
Bringing all this together, we consider the problem of a $v$ type bidder who decides to enter the auction, and seeks to maximize his expected payoff. Following a common mechanism design practice, we understand this problem of the $v$ type bidder as a problem of choosing another type $\tilde{v}$, whose equilibrium strategy the type $v$ wants to imitate and probability of winning and expected inferences by the receiver he wants to obtain, in order to maximize his expected payoff. Thus, given an equilibrium bidding function $\beta$, the problem of a $v$ type bidder is:

$\max _{\tilde{v}}\left\{F^{n-1}(\tilde{v})[v-\beta(\tilde{v})+\tilde{v}]+\int_{\tilde{v}}^{\bar{v}} \frac{1}{F(y)-F(\tau)} \int_{\tau}^{y} x d F(x) d F^{n-1}(y)-\varphi\right\}$.

The first order condition is

$$
\left(F^{n-1}(\tilde{v}) \beta(\tilde{v})\right)^{\prime}=\left(F^{n-1}(\tilde{v})\right)^{\prime}(v+\tilde{v})+F^{n-1}(\tilde{v})-\frac{1}{F(\tilde{v})-F(\tau)} \int_{\tau}^{\tilde{v}} x d F(x)\left(F^{n-1}(\tilde{v})\right)^{\prime} .
$$

Of course, in equilibrium the bidding function must be such that each bidder type strictly prefers his own equilibrium bid to imitating another type, such that we impose $\tilde{v}=v$.

Proposition 1 For a given entry fee $\varphi$ and cut off type $\tau$, the unique fully separating PBE bidding strategy is for all

$$
\beta(v)=v-\frac{F^{n-1}(\tau)}{F^{n-1}(v)} \tau+\frac{1}{F^{n-1}(v)} \int_{\tau}^{v}\left(y-\frac{\int_{\tau}^{y} x d F(x)}{F(y)-F(\tau)}\right) d F^{n-1}(y),
$$

such that $\beta(\tau)=0$ and $\beta^{\prime}(v)$ for all $v \in(\tau, \bar{v})$.

The proof of Proposition 1 first explains that in equilibrium, it must be that $\beta(\tau)=0$, because the $\tau$ type bidders otherwise have a strict incentive to deviate to a zero bid. The proof then derives the equilibrium bidding function and finally demonstrates that this equilibrium satisfies the necessary global strict second order conditions.

We now turn to the bidders' entry decisions. For entry fees that induce entry by only a strict subset of the type space, the bidder type with cut off valuation $\tau$ must be indifferent between paying the entry fee to participate in the auction on one hand, and staying out on the other hand. If the cut off type $\tau$ stays out, he pools with all the nonparticipating lower bidders and obtains a payoff from the receiver's inferences equal to $\frac{\int_{\mathrm{v}}^{\tau} v d F(v)}{F(\tau)}$. If the $\tau$ type decides to pay the entry fee, he wins the auction with probability $F^{n-1}(\tau)$ with a zero bid, in which case he 
obtains the object he vaues $\tau$ and is perceived as type $\tau$ by the receiver. Otherwise, he gets the expected inference of a losing participating bidder

$$
\int_{\tau}^{\bar{v}} \frac{\int_{\tau}^{y} x d F(x)}{F(y)-F(\tau)} d F^{n-1}(y)
$$

Therefore, the equilibrium entry strategies for an interal $\tau \in(\underline{\mathrm{v}}, \bar{v})$ are characterized by the following relationship between the entry fee $\varphi$ and the cut off type $\tau$ :

$$
\varphi=F^{n-1}(\tau) 2 \tau+\int_{\tau}^{\bar{v}} \frac{\int_{\tau}^{y} x d F(x)}{F(y)-F(\tau)} d F^{n-1}(y)-\frac{\int_{\underline{\underline{v}}}^{\tau} y d F(y)}{F(\tau)} .
$$

Hence, the maximal entry fee $\varphi$ that the cut off type $\tau$ is willing to pay is equal to the sum of the expected prize and the difference between the receiver's expected inferences about a participating bidder and a nonparticipating bidder. A quick inspection of equation 5 shows us that, first, the maximal entry fee guaranteeing full participation is

$$
\hat{\varphi}=\int_{\underline{\mathrm{v}}}^{\bar{v}} y-\frac{\int_{\underline{\mathrm{v}}}^{y} F(x) d x}{F(y)} d F^{n-1}(y)-\underline{\mathrm{v}},
$$

second, the lowest fee guaranteeing no participation is

$$
\bar{\varphi}=2 \bar{v}-E(V) \text {, }
$$

i.e., the sum of the inference and prize the $\bar{v}$ type bidder gets with probability 1 if he participates minus what he gets if he pools with all the other non-participating bidders, and, third, $\varphi$ strictly increases with $\tau$ in the interval $[\hat{\varphi}, \bar{\varphi}]$.

This characterization of the equilibrium bidding and entry decisions now allows us to proceed to the final step: what entry fee should the auctioneer choose in order to maximize the auction's expected revenue? The expected revenue of the auction consists of both the expected entry fees paid by the participating bidders and the winner's expected payment:

$$
E R(\tau)=n \varphi(1-F(\tau))+\int_{\tau}^{\bar{v}} \beta(v) d F^{n-1}(v) .
$$

Increasing the entry fee beyond $\hat{\varphi}$ means that the auctioneer collects a higher entry fee from the participating bidders, increases the risk that bidders choose to stay out and decreases the equilibrium bid of all participating bidders. The following Proposition characterizes the optimal entry fee. 
Proposition 2 If all bidders play the fully separating D1 PBE, then the expected revenue is maximal at $\hat{\varphi}$, the maximal entry fee that guarantees full participation.

In order to maximize the auction's expected revenue, the auctioneer does not set the entry fee in a way that allows for a strict subset of (higher) bidder types to distinguish themselves from remaining lower bidder types by participating. Rather, the auctioneer chooses $\varphi$ such that the receiver holds the worst possible beliefs about a nonparticipating bidder. He then fully exploits the bidders' fear of being singled out as such a worst type $\underline{\mathrm{v}}$ for not participating, in order to collect the maximal sum of entry fees from all bidders. Note that this full participation contrasts with the optimal entry fee of the equivalent auction without signaling, where the optimal fee must exclude a part of the bidder types from participation. The proof of Proposition 2 first demonstrates the following result, which is presented here as a Corollary, and which we show to be equivalent to stating Proposition 2 .

Corollary 3 The bidders' ex ante expected payoffs strictly increase with the entry fee $\varphi$, for $\varphi \in[\hat{\varphi}, \bar{\varphi}]$.

A bidder's ex ante payoff consists of his expected prize, $\int_{\underline{v}}^{\bar{v}} F^{n-1}(v) v d F(v)$, the expected inferences of the receiver, and his expected payment as a negative, where the latter consists of the entry fee and the expected value of paying the winner's bid, i.e., $\int_{\underline{\underline{v}}}^{\bar{v}} F^{n-1}(v) \beta(v) d F(v)$.

$$
\begin{aligned}
E U(\tau)= & F(\tau) \frac{\int_{\underline{\mathrm{v}}}^{\tau} y d F(y)}{F(\tau)}+\int_{\tau}^{\bar{v}} F^{n-1}(v)(2 v-\beta(v)) d F(v) \\
& +\int_{\tau}^{\bar{v}} \int_{v}^{\bar{v}} \frac{\int_{\tau}^{y} x d F(x)}{F(y)-F(\tau)} d F^{n-1}(y) d F(v)-(1-F(\tau)) \varphi
\end{aligned}
$$

The receiver's Bayesian beliefs are a martingale, and are thus ex ante independent of $\varphi$. The expected prize decreases with $\varphi$, because increasing $\varphi$ in $[\hat{\varphi}, \bar{\varphi}]$ increases the probability that no bidder will wish to pay the entry fee, and that the object thus remains with the auctioneer. Hence, if the ex ante expected payoff increases with $\varphi$, the receiver's ex ante expected inferences are independent of $\varphi$, and the ex ante expected prize decreases with $\varphi$, then it must be that the ex ante expected payment, and thereby the auction's expected revenue, strictly decreases with $\varphi$.

\section{Discussion}

We have shown that the optimal entry fee in an independent private value first-price auction with signaling is the maximal fee that guarantees 
full participation. What about other auction formats? Bos and Truyts (2014) also investigate the equivalent all-pay, second-price and English auctions with signaling. It seems relevant to consider the role of entry fees in these auction formats as well. For the all-pay auction, this is a straightforward exercise. Considering entry does affect the fact that the signaling incentives and expected inferences of the receiver are identical in the first-price and all-pay auctions. As in Bos and Truyts (2014), the all-pay auction is equivalent to the first-price auction in terms of expected payments and expected revenues, such that the optimal entry fee in the all-pay auction is the same as the optimal fee $\hat{\varphi}$ of the first-price auction mentioned above.

However, introducing entry in the second-price and minimal information English button auctions with signaling, as in Bos and Truyts (2014), proves to be more complicated. The principal reason is that in these auctions, the winning bidder's payment reflects the valuation of the second highest bidder. Thus, the receiver knows that one of the losing bidders has the valuation reflected in the winner's payment, say $v$, while the other participating losing bidders have a valuation between $\tau$ and $v$. However, for $\tau \in(\underline{\mathrm{v}}, \bar{v})$ the number of participating losing bidders depends on the bidders' randomly drawn valuations, such that the receiver's expectation of a losing participating bidder's type, given a second highest type $v$ and a cut off type $\tau$, is:

$\frac{1}{F^{n-2}(v)} \sum_{i=0}^{n-2}\left(\begin{array}{c}n-2 \\ i\end{array}\right) \frac{F^{n-2-i}(\tau)(F(v)-F(\tau))^{i}}{i+1}\left(v+i \frac{\int_{\tau}^{v} y d F(y)}{F(v)-F(\tau)}\right)$.

Of course, a participating bidder does not know ex ante the valuation of the second-highest bidder if this valuation turns out to be higher than his own, and he must consider the expected value of (6) w.r.t. $v$ in order to determine his optimal bidding strategy. As a result, determining the optimal bidding strategy, and even more so the expected revenue, becomes a very tedious exercise. Moreover, the existence of a fully separating equilibrium is far from guaranteed. In Appendix A.3, we explore the bidder's problem in the second-price and English auction with signaling and entry, for the case where $F$ is the uniform distribution over the unit interval. This exercise shows that, for the simple case of the uniform distribution, the problem of finding the optimal entry fee tends to be impossible for the second-price and English auctions, either because of the non-existence of a fully separating equilibrium, or because the equilibrium bidding function is not well-defined. 


\section{References}

Banks, J. S., And J. Sobel (1987): "Equilibrium Selection in Signaling Games," Econometrica, 55, 647-661.

Bos, O., And T. Truyts (2014): "Auctions with Prestige Motives," CORE Discussion Paper 2014/30.

Das VARma, G. (2003): "Bidding for a process innovation under alternative modes of competition," International Journal of Industrial Organization, 21(1), 15-37.

Giovannoni, F., And M. Makris (2014): "Reputational Bidding," International Economic Review, 55(3), 693-710.

Goeree, J. K. (2003): "Bidding for the future: Signaling in auctions with an aftermarket," Journal of Economic Theory, 108(2), 345364 .

Janssen, M. C., V. A. Karamychev, and E. MaAsland (2011): "Auctions with flexible entry fees: A note," Games and Economic Behavior, 72(2), 594-601.

Katzman, B. E., and M. Rhodes-Kropf (2008): "The Consequences of Information Revealed in Auctions," Applied Economics Research Bulletin, 2, 53-87.

Levin, D., And J. L. Smith (1994): "Equilibrium in Auctions with Entry," The American Economic Review, 84(3), 585-599.

\section{A Mathematical Appendix}

\section{A.1 Proof of Proposition 1}

We proceed in 3 steps: 1 ) demonstrating that in a $\mathrm{D} 1 \operatorname{PBE} \beta(\tau)>0$, 2) deriving the bidding function in Proposition 1 and 3) showing that $\beta$ satisfies the necessary second order conditions.

Step 1: Suppose that in equilibrium $\beta(\tau)>0$. Because $\beta$ is a strictly increasing function, the $\tau$ type bidder who pays the entry fee can only win if all other bidders have a valuation strictly smaller than $\tau$. This happens with probability $F^{n-1}(\tau)$. If the $\tau$ type bidder deviation pays the entry fee but deviates to a zero bid, he still wins the auction with probability $F^{n-1}(\tau)$, but in this case no longer pays his strictly positive bid as a winner. A receiver with D1 beliefs attributes such an out-ofequilibrium bid to at least the $\tau$ bidder. Hence, this deviation constitutes a strict improvement for the bidder, such that $\beta(\tau)>0$ is not consistent with a D1 PBE. 
Step 2: From (3), after imposing $\tilde{v}=v$ and rewriting, we obtain

$$
\left(F^{n-1}(v) \beta(v)\right)^{\prime}=\left(F^{n-1}(v) v\right)^{\prime}+\left(F^{n-1}(v)\right)^{\prime} v-\frac{\int_{\tau}^{v} x d F(x)}{F(v)-F(\tau)}\left(F^{n-1}(v)\right)^{\prime}
$$

After integration and using $\beta(\tau)=0$, we obtain

$$
F^{n-1}(v) \beta(v)=\left(F^{n-1}(v) v\right)-F^{n-1}(\tau) \tau+\int_{\tau}^{v} y d F^{n-1}(y)-\int_{\tau}^{v} \frac{\int_{\tau}^{y} x d F(x)}{F(y)-F(\tau)} d F^{n-1}(y),
$$

which, using a few elementary algebraic operations, can be rewritten into the bidding function in Proposition 1.

Step 3. As in Bos and Truyts (2014), we first show that a strictly increasing bidding function implies local strict concavity of the bidder's problem, and, second, that the equilibrium bid is then a global expected utility maximizing choice for each bidder.

First, use the first order condition (3) to define

$$
\begin{gathered}
G(\tilde{v}, v) \equiv\left(F^{n-1}(\tilde{v})\right)^{\prime}(v+\tilde{v})-\left(F^{n-1}(\tilde{v}) \beta(\tilde{v})\right)^{\prime} \\
+F^{n-1}(\tilde{v})-\frac{1}{F(\tilde{v})-F(\tau)} \int_{\tau}^{\tilde{v}} x d F(x)\left(F^{n-1}(\tilde{v})\right)^{\prime}=0
\end{gathered}
$$

which defines $\beta(v)$ for $\tilde{v}=v$. By the implicit function theorem $\beta^{\prime}(v)>0$ if and only if

$$
-\frac{G_{2}(\tilde{v}, v)}{G_{1}(\tilde{v}, v)}=-\frac{\left(F^{n-1}(\tilde{v})\right)^{\prime}}{G_{1}(\tilde{v}, v)}>0
$$

which is only satisfied if $G_{1}(\tilde{v}, v)<0$ for all $v$ at $\tilde{v}=v$. To see that $\beta$ globally maximizes the bidder's problem, note that, by construction, $G(\tilde{v}, v)=0$ is satisfied at $\tilde{v}=v$, while $G_{2}(\tilde{v}, v)>0$ for all $\tilde{v}>v$, such that type $v$ 's utility reaches a unique maximum at $\tilde{v}=v$. Hence, we have that the second order condition is satisfied if $\beta^{\prime}(v)>0$ for all $v \in[\underline{\mathrm{v}}, \bar{v}]$. Note then that:

$$
\beta^{\prime}(v)=1+\frac{(n-1) f(v)}{F(v)}\left(\begin{array}{c}
\frac{F^{n-1}(\tau)}{F^{n-1}(v)} \tau+\left(v-\frac{\int_{\tau}^{v} x d F(x)}{F(v)-F(\tau)}\right) \\
-\frac{1}{F^{n-1}(v)} \int_{\tau}^{v}\left(y-\frac{\int_{\tau}^{y} x d F(x)}{F(y)-F(\tau)}\right) d F^{n-1}(y)
\end{array}\right)>0,
$$

because

$$
\begin{aligned}
& \frac{1}{F^{n-1}(v)} \int_{\tau}^{v}\left(y-\frac{\int_{\tau}^{y} x d F(x)}{F(y)-F(\tau)}\right) d F^{n-1}(y) \\
< & \frac{1}{F^{n-1}(v)-F^{n-1}(\tau)} \int_{\tau}^{v}\left(y-\frac{\int_{\tau}^{y} x d F(x)}{F(y)-F(\tau)}\right) d F^{n-1}(y) \\
< & v-\frac{\int_{\tau}^{v} x d F(x)}{F(v)-F(\tau)} .
\end{aligned}
$$




\section{A.2 Proof of Corollary 3}

Using (4) and (5), we write the expected payoff of a type $v$ bidder who pays $\varphi$ as:

$$
\begin{aligned}
\pi(v) \equiv & F^{n-1}(v)(2 v-\beta(v))+\int_{v}^{\bar{v}} \frac{\int_{\tau}^{y} x d F(x)}{F(y)-F(\tau)} d F^{n-1}(y)-\varphi \\
= & F^{n-1}(v)\left(2 v-v+\frac{F^{n-1}(\tau)}{F^{n-1}(v)} \tau-\frac{1}{F^{n-1}(v)} \int_{\tau}^{v}\left(y-\frac{\int_{\tau}^{y} x d F(x)}{F(y)-F(\tau)}\right) d F^{n-1}(y)\right) \\
& +\int_{v}^{\bar{v}} \frac{\int_{\tau}^{y} x d F(x)}{F(y)-F(\tau)} d F^{n-1}(y)-F^{n-1}(\tau) 2 \tau-\int_{\tau}^{\bar{v}} \frac{\int_{\tau}^{y} x d F(x)}{F(y)-F(\tau)} d F^{n-1}(y)+\frac{\int_{\underline{\mathrm{v}}} \tau d F(y)}{F(\tau)} \\
=v F^{n-1}(v)-F^{n-1}(\tau) \tau-\int_{\tau}^{v} y d F^{n-1}(y)+\int_{\tau}^{v} \frac{\int_{\tau}^{y} x d F(x)}{F(y)-F(\tau)} d F^{n-1}(y) & \\
+\int_{v}^{\bar{v}} & \frac{\int_{\tau}^{y} x d F(x)}{F(y)-F(\tau)} d F^{n-1}(y)-\int_{\tau}^{\bar{v}} \frac{\int_{\tau}^{y} x d F(x)}{F(y)-F(\tau)} d F^{n-1}(y)+\frac{\int_{\underline{\mathrm{v}}}^{\tau} y d F(y)}{F(\tau)} \\
& =v F^{n-1}(v)-F^{n-1}(\tau) \tau-\int_{\tau}^{v} y d F^{n-1}(y)+\frac{\int_{\underline{\mathrm{v}}}^{\tau} y d F(y)}{F(\tau)} \\
& =\int_{\tau}^{v} F^{n-1}(y) d y+\frac{\int_{\underline{\mathrm{v}}}^{\tau} y d F(y)}{F(\tau)}
\end{aligned}
$$

The ex ante average expected payoff of a bidder is then

$$
\begin{aligned}
E U & =\frac{\int_{\underline{\mathrm{v}}}^{\tau} y d F(y)}{F(\tau)} \int_{\underline{\mathrm{v}}}^{\tau} d F(v)+\int_{\tau}^{\bar{v}} \pi(v) d F(v) \\
& =\frac{\int_{\underline{\mathrm{v}}}^{\tau} y d F(y)}{F(\tau)}+\int_{\tau}^{\bar{v}} \int_{\tau}^{v} F^{n-1}(y) d y d F(v) .
\end{aligned}
$$

The derivative w.r.t. $\tau$ then becomes

$$
\begin{aligned}
\frac{\partial E U}{\partial \tau} & =\frac{\tau f(\tau) F(\tau)-f(\tau) \int_{\underline{\underline{v}}}^{\tau} y d F(y)}{(F(\tau))^{2}}+\int_{\tau}^{\tau} F^{n-1}(y) d y F^{n-1}(\tau) \\
& =f(\tau) F(\tau)\left(\tau-\frac{\int_{\underline{v}}^{\tau} y d F(y)}{(F(\tau))}\right)>0 .
\end{aligned}
$$

\section{A.3 Derivations for the second-price and English auctions $^{2}$}

For the second-price auction where $F$ is the uniform distribution over the unit interval and assuming the existence of a strictly increasing equilibrium bidding function, the problem of a type $v$ bidder choosing which

\footnotetext{
${ }^{2} \mathrm{~A}$ more extensive derivation of the results presented in this Section is available from the authors upon simple request.
} 
type $\tilde{v}$ to imitate reads:

$$
\begin{aligned}
& \tilde{v}^{n-1} v-\int_{\tau}^{\tilde{v}} \beta(y) d y^{n-1}+\int_{\tau}^{\tilde{v}} \frac{\int_{x}^{1} y d y}{1-x} d x^{n-1}+\tau^{n-1} \frac{\int_{\tau}^{1} x d x}{1-\tau}-\varphi \\
& +(n-1) \tilde{v}^{n-2}(1-\tilde{v})\left[\frac{1}{\tilde{v}^{n-2}} \sum_{i=0}^{n-2}\left(\begin{array}{c}
n-2 \\
i
\end{array}\right) \frac{\tau^{n-2-i}(\tilde{v}-\tau)^{i}}{i+1}\left(\tilde{v}+i \frac{\int_{\tau}^{\tilde{v}} y d y}{\tilde{v}-\tau}\right)\right] \\
& +\int_{\tilde{v}}^{1} \frac{\sum_{i=1}^{n-2}\left(\begin{array}{c}
n-2 \\
i
\end{array}\right) \frac{\tau^{n-2-i}(y-\tau)^{i}}{i+1}\left(\tilde{v}+i \frac{\int_{\tau}^{y} x d x}{y-\tau}\right) d\left((n-1) y^{n-2}-(n-2) y^{n-1}\right)}{\tilde{v}^{n-2}-\tau^{n-2}} .
\end{aligned}
$$

Deriving the first order condition for $\tilde{v}$, imposing $\tilde{v}=v$ and after a series of algebraic manipulations, we obtain the following bidding function

$$
\begin{aligned}
\beta(v)= & v+\frac{1}{2}-\frac{1}{2}\left(\frac{v}{(n-1)}-\frac{\tau^{n-1}}{v^{n-2}(n-1)}+\tau\right)+\frac{(1-v)}{2}\left(n+\frac{(n-2) \tau}{v}\right) \\
& -\frac{(n-2)(1-v)}{\left(v^{n-2}-\tau^{n-2}\right) v}\left(\frac{v^{n-1}-\tau^{n-1}}{2(n-1)}-\tau^{n-2} v+\frac{v+\tau}{2} v^{n-2}\right) .
\end{aligned}
$$

Note that if $n=3, \beta(v)$ reduces to

$$
\beta(v)=\frac{5-3 \tau}{4}+\frac{(\tau+1) \tau}{4 v},
$$

which decreases with $v$, and thus contradicts the initial assumption of a strictly increasing bidding function, such that a fully separating PBE does not exist for $n=3$. In general, this bidding function tends to be undefined at $\tau$, and this impedes the characterization of the optimal entry fee for the second-price auction.

For the English auction, again assuming a strictly increasing exit rule $\beta$, a type $v$ bidder chooses to exit at the price where the expected payoffs of winning and losing are equal:

$$
\begin{aligned}
& v-b+\frac{\int_{\beta^{-1}(b)}^{1} x d x}{1-\beta^{-1}(b)} \\
= & \frac{1}{\left(\beta^{-1}(b)-\tau\right)^{n-2}} \sum_{i=0}^{n-2}\left(\begin{array}{c}
n-2 \\
i
\end{array}\right)(\tau)^{n-2-i}\left(\beta^{-1}(b)-\tau\right)^{i}\left(\frac{\beta^{-1}(b)+i \frac{\int_{\tau}^{\beta^{-1}(b)} x d x}{\beta^{-1}(b)-\tau}}{i+1}\right),
\end{aligned}
$$

such that the optimal exit strategy can be solved as:

$$
\beta(v)=\frac{1+3 v}{2}-\frac{1}{(v-\tau)^{n-2}}\left(\frac{v^{n-1}-\tau^{n-1}}{2(n-1)}+\frac{v^{n-1}+\tau v^{n-2}}{2}\right)
$$


However, this exit rule can be shown to be non-increasing for a nontrivial subset of the typespace, which contradicts the above assumption of a strictly increasing $\beta$, and demonstrates the non-existence of a fully separating equilibrium. For instance, for $n=3, \beta(v)$ reduces to

$$
\beta(v)=\frac{\left(3 v^{2}-8 v \tau+2 v+\tau^{2}-2 \tau\right)}{4(v-\tau)},
$$

which is nonmonotonic with respect to $v$ on $[\tau, 1]$. For $n=4, \beta(v)$ becomes

$$
\beta(v)=\frac{1+3 v}{2}-\frac{1}{(v-\tau)^{2}}\left(\frac{v^{3}-\tau^{3}}{6}+\frac{v^{3}+\tau v^{2}}{2}\right),
$$

which is equally nonmonotonic w.r.t. $v$ on $[\tau, 1]$. 